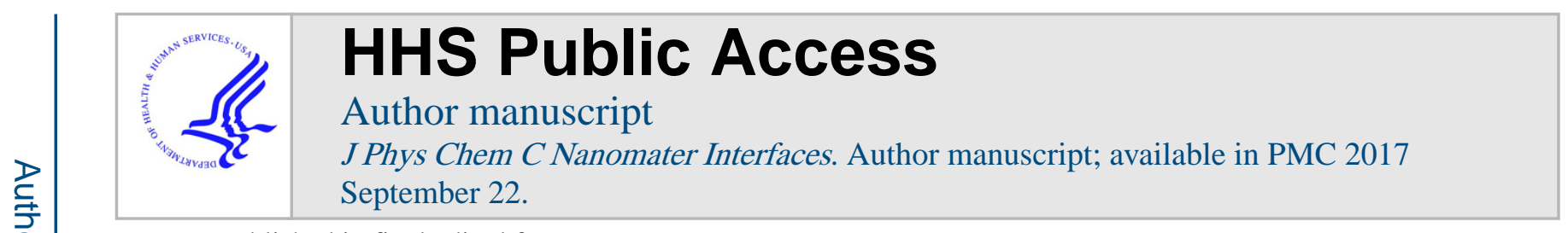

Published in final edited form as:

J Phys Chem C Nanomater Interfaces. 2016 September 22; 120(37): 20555-20562. doi:10.1021/acs.jpcc. 5 b11702.

\title{
Harnessing Leaky Modes for Fluorescence Enhancement in Gold-Tipped Silicon Nanowires
}

\author{
Xin Zhao ${ }^{\dagger, \ddagger}$, M. H. Alizadeh ${ }^{\ddagger}, \S$, and Björn M. Reinhard ${ }^{\dagger, \ddagger},{ }^{*}$ \\ tDepartment of Chemistry, Boston University, Boston, Massachusetts 02215, United States

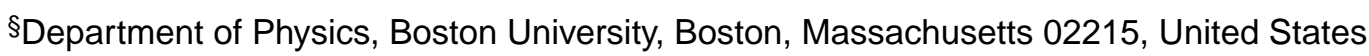 \\ ¥The Photonics Center, Boston University, Boston, Massachusetts 02215, United States
}

\begin{abstract}
Hybrid structures containing plasmonic and photonic components can enhance light-matter interaction through both photonic and plasmonic modes and potentially their interactions. In this article, we systematically investigate the optical properties of nanoparticle-tipped silicon nanowires (NPTWs) and characterize their ability to enhance the fluorescence signal of two cyanine dyes (Cy3 and $\mathrm{Cy} 5$ ) selectively bound to the gold tip. Although both gold and silicon components contribute to the fluorescence signal enhancement, the experimentally observed strong morphology dependence of the fluorescence emission enhancement is demonstrated to depend on tunable leaky modes of the silicon nanowires. The ability to encode emission fluorescence enhancement in the structure of NPTWs is of high relevance for multiparametric biosensing and imaging.
\end{abstract}

\section{Graphical Abstract}
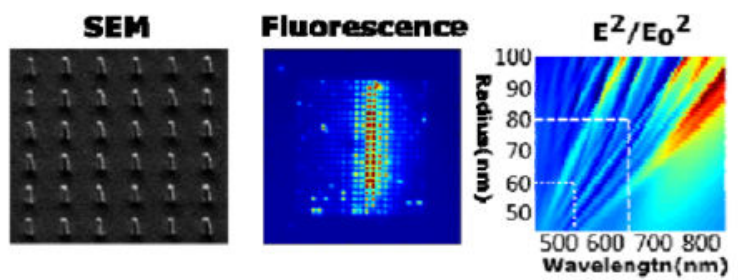

\section{Introduction}

Silicon wires represent photonic resonators and waveguides whose optical properties are tunable by the dimensions of the wire. ${ }^{1-5}$ The ability to enhance light-matter interactions in and around nanoscale silicon nanowires has attracted significant interest in these materials

*Corresponding Author: bmr@bu.edu.

The authors declare no competing financial interest.

ASSOCIATE CONTENT

Supporting Information

The Supporting Information is available free of charge on the ACS Publication website at DOI: 10.1021/acs.jpcc.5b11702

Fluorescence intensity maps and FDTD simulation results (PDF) 
for various applications in areas ranging from light harvesting ${ }^{2,6-7}$ to biosensing. ${ }^{8-11}$ One application of particular note is the use of silicon nanowires decorated with metallic particles as tunable electromagnetic substrates for surface-enhanced Raman spectroscopy (SERS). ${ }^{12-18}$ The general idea underlying this approach is that electromagnetic modes of the silicon nanowire can hybridize with those of the plasmonic component to shape the nearfield around the hybrid structure in both spatial and frequency domains. ${ }^{19}$ The electromagnetic modes of very long nanowires with radius $R$ can be found by solving the dispersion relation ${ }^{20-21}$ :

$\left[\frac{\mu_{c}}{k_{c} R} \frac{J_{m}^{\prime}\left(k_{c} R\right)}{J_{m}\left(k_{c} R\right)}-\frac{\mu}{k R} \frac{H_{m}^{\prime}\left(k_{c} R\right)}{H_{m}\left(k_{c} R\right)}\right] \times\left[\frac{\varepsilon_{c}}{k_{c} R} \frac{J_{m}^{\prime}\left(k_{c} R\right)}{J_{m}\left(k_{c} R\right)}-\frac{\varepsilon}{k R} \frac{H_{m}^{\prime}\left(k_{c} R\right)}{H_{m}\left(k_{c} R\right)}\right]=m^{2} \frac{\left(k_{z} R\right)^{2}}{(\omega R / c)^{2}}\left[\frac{1}{(k R)^{2}}-\frac{1}{\left(k_{c} R\right)^{2}}\right]^{2}$

where $m$ is an integer, $J_{\mathrm{m}}$ and $H_{\mathrm{m}}$ are cylindrical Bessel and Hankel functions, respectively, $k_{\mathrm{c}}$ is the transverse wavevector component of a mode propagating along the long cylinder axis with a wavevector component $\mathrm{k}_{\mathrm{z}}$, and $k$ is the transverse component of the wavevector in free space. $\mu_{\mathrm{c}}, \mu$ and $\varepsilon_{\mathrm{c}}, \varepsilon$ are the permeabilities and permittivies of the cylinder (labeled with index c) and the medium. Depending on the nature of the transverse wavevector of a mode, it can be categorized as either leaky or guided. A purely imaginary transverse wavevector results in a real wavevector along the cylinder axis, hence resembling a propagating mode confined to the interior of the wire. A real transverse wavevector, however, leads to modes, which leak out of the nanowire. When $\mathrm{m}=0$, transverse magnetic $\mathrm{TM}_{0 l}$ and transverse electric $\mathrm{TE}_{01}$ modes exist independently with radial mode distributions of order 1 . For very thin nanowires only the fundamental $\mathrm{HE}_{11}$ mode is excited. This mode shows large modal delocalization to the surrounding of the nanowire and has no cutoff frequency. This mode is illustrated in Figure 1a, where its significant spillover into the surrounding of the nanowire is evident. Also the mode intensity for higher modes of a silicon nanowire with a radius of $150 \mathrm{~nm}$ and length of $3 \mu \mathrm{m}$ under an x polarized incident light is shown in Figure 1. The gold nanoparticle on the NPTWs allows a selective functionalization of the tip with fluorescence sensors due to the high affinity of thiols to gold, providing excellent spatial control of the positioning of the emitter. Furthermore, excitation of localized surface plasmon resonances (LSPRs) of the gold nanoparticles through photonic modes of the silicon wire results in field localization around the metal tip $^{22}$. Both LSPRs and photonic resonances of the silicon nanowire provide enhanced density of optical states that can boost the radiative decay rates of near-field coupled quantum emitters. ${ }^{23-26}$ The aim of this article is to characterize these interactions through systematic analysis of the morphology dependence of the fluorescence intensity obtained for dye-functionalized NPTWs. We will, thus, elucidate the underlying working principles of fluorescence enhancement in these electromagnetic hybrid materials and derive useful design criteria for maximizing their performance. 


\section{Methods}

\section{Fabrication of NPTW Array}

The A3 poly(methyl methacrylate) (PMMA) ( (molecular weight 950000) was first spincoated on $1 \mathrm{~cm} \times 1 \mathrm{~cm}$ piranha cleaned silicon substrates at a speed of $2000 \mathrm{rpm}$ for $45 \mathrm{~s}$ to reach a final thickness of approximately $180 \mathrm{~nm}$. The substrates were subsequently softbaked at $170^{\circ} \mathrm{C}$ for $15 \mathrm{~min}$ and then sputtered with $10 \mathrm{~nm}$ gold film. Periodic nanoholes of radius ranging from 50 to $100 \mathrm{~nm}$ were then patterned through EBL by Zeiss SUPRA 40VP SEM equipped with a Ratin beam blanker and a nanopattern generation system (NPGS). After chemical etching of the gold film and development in a methyl isobutyle ketone $($ MIBK)/isopropanol (IPA) =1:3 solution, a well-defined structure in PMMA was obtained. $\mathrm{A} \mathrm{Cr} / \mathrm{Au} / \mathrm{Al}$ layer with a thickness of 5/80/40 nm, was then deposited on the exposed EBLpatterned surface by electron beam evaporation (CHA Industries, Fermon, CA, U.S.A.) with deposition rates of $0.5 / 1.0 / 1.0 \AA / \mathrm{s}$ for each metal to create a hybrid material with a gold tip on top. The Cr layer was used to increase the adhesion between gold and the silicon surface and the aluminum layer was deposited to protect the gold layer from anisotropic dry etch gases. After the PMMA layer was removed by immersion in acetone at room temperature overnight followed by sonication in a fresh acetone bath, substrates were ready for reactive ion etching (RIE). We used the inductively coupled plasma reactive ion etching (ICP-RIE, STS company) at Harvard University with a gas mixture of $\mathrm{SF}_{6} / \mathrm{C}_{4} \mathrm{~F}_{8}(60 / 160 \mathrm{sccm})$ at a power of $1200 \mathrm{~W}$ for $6-8 \mathrm{~min}$ to reach a final height of $\sim 1 \mu \mathrm{m}$. During the etching, SF6 and C4F8 gases were used for the etching and surface passivation respectively. The RIE chamber was cleaned with $\mathrm{O}_{2}$ for $20 \mathrm{~min}$ and conditioned run with the etching gases for $10 \mathrm{~min}$ prior to the RIE process on samples to ensure reproducible etching results. Dummy samples were placed in the chamber every time before etching of real samples to determine the etching rate with a profilometer. After etching, the aluminum masks were removed using an aluminum etchant-type A (Transene) and rinsed thoroughly with DI water. SEM images were taken on a Zeiss SUPRA55.

\section{Fluorescence Microscopy}

Fluorescence images of the NPTW arrays and gold disk controls were obtained using an inverted microscope equipped with a high-resolution electron multiplying charge coupled detector (EMCCD). A mercury lamp in combination with appropriate filters were used as the illumination source throughout the whole measurement and a long working distance objective $60 \times$ air $(\mathrm{NA}=0.7)$ was used to acquire the image.

\section{Spectroscopic Characterization}

Scattering measurements were carried out on silicon nanowires with different radii. The incident light was focused using a $50 \times$ air objective $(\mathrm{NA}=0.75)$ with the backscattered light collected by the same objective. The obtained spectra were background corrected and finally normalized by the spectrum acquired with $200 \mathrm{~nm}$ polystyrene bead by drying the polystyrene bead on silicon. 


\section{Characterization of the Numerical Methods}

The silicon nanowire and NPTW optical properties were simulated using the finite difference time domain (FDTD) solver of Lumerical Solution, Inc. For scattering spectra and near-field simulation, total-field-scattered-field (TFSF) source was used to illuminate the structure with perfectly matched layer (PML) boundary conditions imposed in all planes. A box monitor was placed surrounding the source region to compute the scattered fluxes as a function of wavelength. A 2D plane monitor was placed $10 \mathrm{~nm}$ above the structure's upper surface to compute the field distribution. In the Purcell factor (PF) simulation, we used an electrical dipole emitter to mimic the dye molecule. The PF was obtained by calculating the emitted power in the presence of the silicon nanowires and normalizing it to the power emitted in vacuum. This ratio represents the PF of the studied system.

\section{Results and Discussion}

NPTWs contain a gold nanoparticle on the tip of a high refractive index silicon wire. We fabricated geometrically well-defined NPTWs through the top-down fabrication strategy ${ }^{27-28}$ outlined in Figure 2a-f. After a mask of metal nanoparticles was generated by electron beam lithography (EBL), anisotropic dry etching was used to create regular arrays of high aspect ratio NPTWs with defined morphology $(R=50-100 \mathrm{~nm}$ with an increment of $5 \mathrm{~nm} ; \mathrm{L}=1 \mu \mathrm{m})$. During the RIE process, the diameter of the EBL written disks decreased somewhat. As this effect applies to all nanowires, the difference in $R$ between two adjacent nanowires remained constant at $5 \mathrm{~nm}$. The separation between the individual posts was close to $1 \mu \mathrm{m}$ to avoid diffractive coupling between the individual wires. Figure $2 \mathrm{~g}$ shows a scanning electron microscope (SEM) image of a NPTW array ( $30^{\circ}$ tilt angle) and illustrates the excellent shape uniformity of the NPTWs. The insets of Figure $2 \mathrm{~g}$ contain magnified SEM images of the interface between gold nanoparticle and silicon nanowire for two different radii. Although nanowires with small radii exhibit some "rounding" of the top surface of the gold nanodisk, as shown in the upper right inset in Figure 2g, the gold-silicon interface remains intact.

After removal of the aluminum mask through a wet etching step, a monolayer of 30 base pairs long thiolated and dye-modified double-stranded DNA was self-assembled selectively on the gold tips ${ }^{29}$, utilizing the high affinity of the thiol residue to the gold nanoparticle. The DNA strands served in the experiments both as a linker and spacer between the gold nanoparticle surface and the fluorescent dyes to prevent a quenching of the dye in close proximity of the metal surface ${ }^{30-31}$. Thiolated DNA assembles on the gold surface in a brush that creates a dense DNA layer between the dyes and the gold surface. Treating the short DNAs as rods due to the long persistence length of double stranded DNA of $53 \mathrm{~nm}$, we estimate that the DNA spacer is approximately $9.7 \mathrm{~nm}$ in length.

$\mathrm{Cy} 3$ and $\mathrm{Cy} 5$ were chosen as representative fluorescent dyes with emission maxima in the green and red. Detailed excitation and emission spectra for both dyes in solution are given in Figure 3a. The quantum yields of Cy3 and Cy 5 are 0.15 and $0.30^{32}$, respectively, which leaves sufficient room for engineering a measurable fluorescence enhancement through custom-designed photonic-plasmonic hybrid structures. 
After establishing a reliable fabrication strategy for well-defined NPTWs, we characterized the fluorescence intensity of Cy3- or Cy5-functionalized NPTWs as function of $R$. All fluorescence measurements were performed with an inverted microscope in epi-mode as shown in Figure 3b. As the silicon substrate is non-transparent, the samples were mounted with the pillars pointing towards the objective for their characterization. Figure $3 \mathrm{c}$ and $\mathrm{d}$ show fluorescence images of NPTW arrays functionalized with Cy3 or Cy5. Cy3 (Cy5) was excited close to the absorption peak at $535 \mathrm{~nm}(650 \mathrm{~nm})$ and its fluorescence was collected at $585 \mathrm{~nm}(680 \mathrm{~nm})$ using proper fluorescence imaging filter sets. The corresponding fluorescence intensities are histogrammed in Figure 3e and f, respectively. Importantly, both $\mathrm{Cy} 3$ and $\mathrm{Cy} 5$ show strongly $R$-dependent fluorescence intensities. The $\mathrm{Cy} 3$ signal is highest for NPTWs with $\mathrm{R} \approx 60 \mathrm{~nm}$ while Cy5 shows a maximum for $\mathrm{R} \approx 80 \mathrm{~nm}$. We also evaluated the fluorescence intensities of the NPWTs in direct comparison with patterned dyes under otherwise identical conditions (Supporting Information, Figure S1). The NPTWs achieved significantly higher fluorescence intensities, consistent with a quantitative fluorescence enhancement.

In the visible range of the electromagnetic spectrum, the non-transparent nature of the silicon substrate is detrimental for many potential applications. However, this problem can be circumvented by transferring the NPTWs into a transparent substrate as has been recently shown for conventional silicon posts. ${ }^{33}$ We verified that these transparent NPTW arrays retain the same fluorescence properties. To that end, NPTWs generated on a silicon support were embedded in polydimethylsiloxane (PDMS), cleaved from the substrate using a razor blade, and finally transferred to a quartz wafer. A schematic drawing of the resulting NPTW array is depicted in Figure 4a. We repeated the fluorescence measurements with these transparent NPTW containing films (Figure 4b-e). For NPTWs embedded in PDMS and transferred to a quartz support we collected the fluorescence signal from the substrate side (Figure 4a). Importantly, although for smaller radii the relative intensities on silicon and quartz show some discrepancies, the fluorescence intensity maxima are identical in the case of Cy5 and very similar for $\mathrm{Cy} 3$.

It is well known that the excitation of the gold nanoparticle plasmon resonance provides a substantial local $E$-field enhancement ${ }^{34-35}$ and that the resonance generates high local density of optical states. ${ }^{36-38}$ Both of these effects can boost the signal intensity of fluorescent dyes localized at the correct separation from the metal nanoparticle surface $^{35,39-42}$. To determine the contribution from the gold nanoparticles to the fluorescence signal enhancement, we measured the fluorescence intensity of the dyes bound to gold nanoparticles of different radii fabricated on a silicon substrate (no nanowire component) (Figure 5). The nanoparticle radius was varied across the same range as the $R$ values for the NPTWs. The elastic scattering intensities of the gold nanoparticles show the expected increase as function of increasing radius (Figure 5a), but the fluorescence signal intensities (Figure $5 \mathrm{~b}$ and $\mathrm{c}$ ) do not exhibit a pronounced radius dependence. The $\mathrm{Cy} 3$ functionalized array (Figure 5b) shows a higher fluorescence intensity than the Cy5 containing array (Figure 5c). This is intriguing considering that the quantum yield of Cy5 is higher than that of $\mathrm{Cy} 3 .{ }^{43}$ Interestingly, the $\mathrm{Cy} 3$ excitation and emission bands overlap with the quadrupolar plasmon resonance of the gold nanoparticles on the silicon substrate (Detailed simulation results can be found in Supporting Information Figure S2), but Cy5 
lacks any comparable overlap with a plasmon resonance. ${ }^{44}$ Although the quadrupolar mode in nanoparticles that are much smaller than the wavelength of light is dark so that dissipative losses dominate, for larger nanoparticles and for high ambient effective refractive indices higher angular modes can radiate. We attribute, consequently, the much higher fluorescence intensity for $\mathrm{Cy} 3$, when compared to $\mathrm{Cy} 5$, to a quadrupolar mode mediated fluorescence emission enhancement. As the quadrupolar mode shows an even weaker size dependence as the dipolar mode see ref ${ }^{45}$ and Supporting Information Figure S2, the Cy3 fluorescence is enhanced for all investigated radii. The slightly higher intensity in the central region of the array in Figure $5 \mathrm{~b}$ is the result of multiple scattering events of emitted light in the presence of neighboring gold nanoparticles. For nanoparticles located at the periphery of the array, multiple scattering is less effective and the signal is overall lower.

From the comparison of the fluorescence intensities of the NPTWs and the gold nanoparticles, we conclude that the strong $R$-dependence observed for the NPTWs is a photonic effect caused by the silicon nanowire component. Photonic modes in silicon nanowires generate enhanced density of states and thus, according to Fermi's Golden Rule, boost the radiative decay rate. The metal nanoparticles still affect the fluorescence enhancement but the effect is more subtle. The broad enhancement of $\mathrm{Cy} 3$ for all $R$ values can account for the overall weaker $R$-dependence for this dye. Since the signal of $\mathrm{Cy} 3$ is already increased by the metal nanoparticle, the amplitude of the gain created by the silicon nanowire is smaller.

After identifying the silicon nanowire as main source for the strong fluorescence signal modulation, we set out to correlate the observed relative fluorescence enhancement with photonic modes of the silicon nanowires. First, we measured the scattering spectra (Figure 6a) of NPTW arrays in an optical microscope using an objective with a numerical aperture of NA $=0.75$, corresponding to a maximum incident angle of $50^{\circ}$. The spectra show two distinct features that continuously red-shift with increasing $R$. To determine the origin of these photonic modes, we simulated the scattering spectra by numerical electromagnetic simulations using the finite difference time domain (FDTD) method (Figure 6b). We assumed an oblique angle of incidence of $\theta=45^{\circ}$ to closely match the experimental conditions (Figure 6c). The global maxima in the experimental spectra are assigned to the $\mathrm{HE}_{11}$ mode due to the excellent agreement of experimental and simulated peak positions as function of silicon nanowire radius as shown in Figure 6d. The higher energy feature in the experimental spectra cannot be assigned to any individual mode and is therefore attributed to mode hybridization.

In the next step, we analyzed the effect that placing a metal nanoparticle on the tip of a silicon nanowire has on the local E-field. Figure 7 compares the FDTD-simulated near field spectra as function of $R$ for silicon nanowires and NPTWs. The near-fields were evaluated $10 \mathrm{~nm}$ above the surface of the respective nanowire. Both structures were excited with a plane wave at an angle of incidence of $90^{\circ}$.

In Figure $7 \mathrm{a}$ and $\mathrm{b}$, we plot the E-field intensity spectra for the silicon nanowire and NPTW as a function of radius. Both maps have the same scale. The comparison of Figure 7a and $b$ 
reveals an enhancement of the $E$-field intensity generated by the photonic modes for wavelengths $<700 \mathrm{~nm}$ due to the excitation of metal nanoparticle plasmons.

We marked the E-field intensities for the $\mathrm{Cy} 3$ (dotted line) and Cy5 (dashed line) excitation wavelengths of 530 and $630 \mathrm{~nm}$ for the $R=60$ and $80 \mathrm{~nm}$ NPTWs in Figure 7a and b. The peak excitation wavelengths lie close to local maxima in the NPTW $E$-field intensity plots and confirm that the NPTW configurations that yield the highest fluorescence intensities in the experiments provide some excitation enhancement. Figure 7c compares the $E$-field intensity maps for a silicon nanowire and a NPTW at the Cy5 excitation wavelength of 637 $\mathrm{nm}$. It is evident that the metal nanoparticle on top of the silicon nanowire efficiently localizes the field around the tip of the wire where the fluorescence molecules are located. Nanoparticle-mediated localization of the $E$-field on the silicon nanowire tips is, however, insufficient to account for the observed $R$-dependence of the fluorescence signal. For instance, for the Cy5 peak excitation wavelength of $630 \mathrm{~nm}$, multiple $R$ values show increased near-field intensity, yet we obtain a strong fluorescence signal exclusively at $R=$ $80 \mathrm{~nm}$. The photonic modes of the silicon nanowire are strongly morphology-dependent ${ }^{46-48}$ and therefore provide ample opportunity for modulating the radiative rates of dye emitters in close proximity to the NPTW. We, consequently, calculated the PF for emitters in the presence of the silicon nanowires to determine the modes involved in the enhancement process. The $\mathrm{PF}$ is a measure of the spontaneous emission rate enhancement due to an enhanced density of states to which the emitter can couple. Further details regarding the simulations are provided in the Methods section. The emitter was placed $20 \mathrm{~nm}$ above the nanowire at an angle of $45^{\circ}$ with respect to the long nanowire axis to accommodate coupling to modes with different symmetries. For instance, only a horizontal dipole can excite the $\mathrm{HE}_{11}$ mode. To account for different locations around the metal nanoparticle we considered an on-axis as well as an off-axis location of the emitter. The dipole emitter was positioned in the center and on the edge of the upper surface of the structure for these two cases, respectively. The resulting PFs at these two locations are plotted as function of wavelength and $R$ in Figure $8 \mathrm{a}$ and $\mathrm{b}$, respectively. The PF plots in Figure 8 resemble in their shape the scattering spectra in Figure $6 \mathrm{c}$, confirming that the $\mathrm{HE}_{11}$ and $\mathrm{TM}_{11}$ modes that already dominated the scattering spectra also result in the strongest PF enhancement. We marked the $R$ values associated with the maximum fluorescence intensity for Cy3 (dotted line) and Cy5 (dashed line) at the respective emission wavelength in Figure 8b. Both of these points lie close to local maxima in the PF plot, confirming that the PF plot provides an accurate prediction of the Cy3, Cy5 fluorescence maxima. Specifically, the Cy3 emitter couples to the leaky $\mathrm{TM}_{11}$ mode, whereas $\mathrm{Cy} 5$ couples to the leaky $\mathrm{HE}_{11}$ mode.

A comparison of the PF plots for the two different positions in Figure 8a and $\mathrm{b}$ allows validation of the robustness of the PF calculations with regard to the emitter position. The overall spectral behavior of the PF is virtually unchanged while the higher numerical values for the off-axis case can be rationalized in terms of edge effects.

The PF plots in Figure 8 exhibit an overall closer correspondence with the experimentally observed $R$ dependence than the scattering plot in Figure $6 \mathrm{~b}$. In PF simulations, similar to the experiment, a near-field emitter couples to the leaky nanowire modes, which in turn leads to a higher local density of EM states in the emitter's position. The scattering cross 
section, however, was calculated under plane wave illumination, which is less similar to the actual experimental condition. We emphasize, however, that despite slight discrepancies in the exact spectral position of the modes in scattering spectra and PF plots in both cases, leaky modes with very similar dispersion properties are excited.

Last but not least, we would like to stress that the operational range of the NPTWs is not limited to the spectral range of the $\mathrm{Cy} 3, \mathrm{Cy} 5$ dyes investigated in this work. The $R-$ dependence of the fluorescence enhancement through NPTWs facilitates an expansion further into the red by increasing the diameter of the nanowires.

\section{Conclusion}

Photonic-plasmonic hybrid structures comprising a gold nanoparticle located on a silicon nanowire with different radii were fabricated and characterized through optical spectroscopy and electromagnetic simulations. After a selective functionalization of the gold tip with Cy3or Cy5-conjugated DNA, the NPTWs showed a strongly radius $(R)$-dependent fluorescence signal enhancement. FDTD simulation of the $E$-field intensity and PF enhancement of the hybrid structures revealed that the experimentally observed stringent morphology dependence is determined by the distinct $R$-dependence of the density of optical states provided by leaky modes of the silicon nanowires. The leaky modes also have large mode volumes compared to plasmon resonances resulting in large medium penetration, albeit at the cost of reduced field strength. NPTWs combine field localization and enhancement at the metallic tip with the superb tunability of photonic modes provided by the silicon nanowires. The greatly improved tunability of the fluorescence signal achieved with the gold nanoparticle-silicon nanowire hybrid when compared with bare nanoparticle paves the path to a wide range of biosensing and imaging applications.

\section{Supplementary Material}

Refer to Web version on PubMed Central for supplementary material.

\section{Acknowledgments}

This work was supported by the National Institutes of Health (NCI) through grant 5R01CA138509 to BMR.

\section{References}

1. Cao L, Fan P, Barnard ES, Brown AM, Brongersma ML. Tuning the Color of Silicon Nanostructures. Nano Lett. 2010; 10:2649-2654. [PubMed: 20507083]

2. Cao L, Fan P, Vasudev AP, White JS, Yu Z, Cai W, Schuller JA, Fan S, Brongersma ML. Semiconductor Nanowire Optical Antenna Solar Absorbers. Nano Lett. 2010; 10:439-445. [PubMed: 20078065]

3. Fountaine KT, Whitney WS, Atwater HA. Resonant Absorption in Semiconductor Nanowires and Nanowire Arrays: Relating Leaky Waveguide Modes to Bloch Photonic Crystal Modes. J Appl Phys. 2014; 116:153106.

4. Walker BN, Stolee JA, Pickel DL, Retterer ST, Vertes A. Tailored Silicon Nanopost Arrays for Resonant Nanophotonic Ion Production. J Phys Chem C. 2010; 114:4835-4840.

5. Seo K, Wober M, Steinvurzel P, Schonbrun E, Dan Y, Ellenbogen T, Crozier KB. Multicolored Vertical Silicon Nanowires. Nano Lett. 2011; 11:1851-1856. [PubMed: 21413684] 
6. Fan G, Zhu H, Wang K, Wei J, Li X, Shu Q, Guo N, Wu D. Graphene/Silicon Nanowire Schottky Junction for Enhanced Light Harvesting. ACS applied materials \& interfaces. 2011; 3:721-725. [PubMed: 21323376]

7. Peng K, Xu Y, Wu Y, Yan Y, Lee ST, Zhu J. Aligned Single-Crystalline Si Nanowire Arrays for Photovoltaic Applications. small. 2005; 1:1062-1067. [PubMed: 17193395]

8. Kandziolka M, Charlton JJ, Kravchenko II, Bradshaw JA, Merkulov IA, Sepaniak MJ, Lavrik NV. Silicon Nanopillars as a Platform for Enhanced Fluorescence Analysis. Anal Chem. 2013; 85:90319038. [PubMed: 23984845]

9. Patolsky F, Timko BP, Yu G, Fang Y, Greytak AB, Zheng G, Lieber CM. Detection, Stimulation, and Inhibition of Neuronal Signals with High-Density Nanowire Transistor Arrays. Science. 2006; 313:1100-1104. [PubMed: 16931757]

10. Gao Z, Agarwal A, Trigg AD, Singh N, Fang C, Tung CH, Fan Y, Buddharaju KD, Kong J. Silicon Nanowire Arrays for Label-Free Detection of DNA. Anal Chem. 2007; 79:3291-3297. [PubMed: 17407259]

11. Li Z, Chen Y, Li X, Kamins T, Nauka K, Williams RS. Sequence-Specific Label-Free DNA Sensors Based on Silicon Nanowires. Nano Lett. 2004; 4:245-247.

12. Wells SM, Merkulov IA, Kravchenko II, Lavrik NV, Sepaniak MJ. Silicon Nanopillars for FieldEnhanced Surface Spectroscopy. Acs Nano. 2012; 6:2948-2959. [PubMed: 22385359]

13. Hatab NA, Hsueh CH, Gaddis AL, Retterer ST, Li JH, Eres G, Zhang Z, Gu B. Free-Standing Optical Gold Bowtie Nanoantenna with Variable Gap Size for Enhanced Raman Spectroscopy. Nano Lett. 2010; 10:4952-4955. [PubMed: 21090585]

14. Wells SM, Polemi A, Lavrik NV, Shuford KL, Sepaniak MJ. Efficient Disc on Pillar Substrates for Surface Enhanced Raman Spectroscopy. Chem Commun. 2011; 47:3814-3816.

15. Zhang B, Wang H, Lu L, Ai K, Zhang G, Cheng X. Large-Area Silver-Coated Silicon Nanowire Arrays for Molecular Sensing Using Surface-Enhanced Raman Spectroscopy. Adv Funct Mater. 2008; 18:2348-2355.

16. Qiu T, Wu X, Shen J, Ha PC, Chu PK. Surface-Enhanced Raman Characteristics of Ag Cap Aggregates on Silicon Nanowire Arrays. Nanotechnology. 2006; 17:5769.

17. He Y, Su S, Xu T, Zhong Y, Zapien JA, Li J, Fan C, Lee ST. Silicon Nanowires-Based HighlyEfficient Sers-Active Platform for Ultrasensitive DNA Detection. Nano Today. 2011; 6:122-130.

18. Han X, Wang H, Ou X, Zhang X. Highly Sensitive, Reproducible, and Stable Sers Sensors Based on Well-Controlled Silver Nanoparticle-Decorated Silicon Nanowire Building Blocks. J Mater Chem. 2012; 22:14127-14132.

19. Polemi A, Wells SM, Lavrik NV, Sepaniak MJ, Shuford KL. Local Field Enhancement of Pillar Nanosurfaces for Sers. J Phys Chem C. 2010; 114:18096-18102.

20. Paniagua-Dominguez R, Grzela G, Rivas JG, Sanchez-Gil J. Enhanced and Directional Emission of Semiconductor Nanowires Tailored through Leaky/Guided Modes. Nanoscale. 2013; 5:1058210590. [PubMed: 24057037]

21. Stratton, JA. Electromagnetic Theory. John Wiley \& Sons; 2007.

22. Coronado EA, Encina ER, Stefani FD. Optical Properties of Metallic Nanoparticles: Manipulating Light, Heat and Forces at the Nanoscale. Nanoscale. 2011; 3:4042-4059. [PubMed: 21931921]

23. Schuller JA, Barnard ES, Cai W, Jun YC, White JS, Brongersma ML. Plasmonics for Extreme Light Concentration and Manipulation. Nat Mater. 2010; 9:193-204. [PubMed: 20168343]

24. Muskens O, Giannini V, Sanchez-Gil J, Gómez Rivas J. Strong Enhancement of the Radiative Decay Rate of Emitters by Single Plasmonic Nanoantennas. Nano Lett. 2007; 7:2871-2875. [PubMed: 17683156]

25. Sauvan C, Hugonin JP, Maksymov I, Lalanne P. Theory of the Spontaneous Optical Emission of Nanosize Photonic and Plasmon Resonators. Phys Rev Lett. 2013; 110:237401. [PubMed: 25167528]

26. Sun G, Khurgin JB, Soref RA. Practicable Enhancement of Spontaneous Emission Using Surface Plasmons. Appl Phys Lett. 2007; 90:111107.

27. Thiruvengadathan R, Korampally V, Ghosh A, Chanda N, Gangopadhyay K, Gangopadhyay S. Nanomaterial Processing Using Self-Assembly-Bottom-up Chemical and Biological Approaches. Rep Prog Phys. 2013; 76:066501. [PubMed: 23722189] 
28. Wang X, Ocola LE, Divan RS, Sumant AV. Nanopatterning of Ultrananocrystalline Diamond Nanowires. Nanotechnology. 2012; 23:075301. [PubMed: 22261094]

29. Ahn W, Hong Y, Boriskina SV, Reinhard BrM. Demonstration of Efficient on-Chip Photon Transfer in Self-Assembled Optoplasmonic Networks. ACS nano. 2013; 7:4470-4478. [PubMed: 23600526]

30. Dulkeith E, Morteani A, Niedereichholz T, Klar T, Feldmann J, Levi S, Van Veggel F, Reinhoudt D, Möller M, Gittins D. Fluorescence Quenching of Dye Molecules near Gold Nanoparticles: Radiative and Nonradiative Effects. Phys Rev Lett. 2002; 89:203002. [PubMed: 12443474]

31. Dulkeith E, Ringler M, Klar T, Feldmann J, Munoz Javier A, Parak W. Gold Nanoparticles Quench Fluorescence by Phase Induced Radiative Rate Suppression. Nano Lett. 2005; 5:585-589. [PubMed: 15826091]

32. [accessed 03/01/2016] Cyanine Dyes: Cy3: Cy5 and Others. http://www.atdbio.com/content/32/ Cyanine-dyes-Cy3-Cy5-andothers

33. Park H, Seo K, Crozier KB. Adding Colors to Polydimethylsiloxane by Embedding Vertical Silicon Nanowires. Appl Phys Lett. 2012; 101:193107.

34. Radko IP, Volkov VS, Beermann J, Evlyukhin AB, Søndergaard T, Boltasseva A, Bozhevolnyi SI. Plasmonic Metasurfaces for Waveguiding and Field Enhancement. Laser \& Photonics Reviews. 2009; 3:575-590.

35. Ding P, Liang E, Hu W, Cai G, Xue Q. Tunable Plasmonic Properties and Giant Field Enhancement in Asymmetric Double Split Ring Arrays. Photonics and Nanostructures-Fundamentals and Applications. 2011; 9:42-48.

36. Lozano G, Louwers DJ, Rodríguez SR, Murai S, Jansen OT, Verschuuren MA, Rivas JG. Plasmonics for Solid-State Lighting: Enhanced Excitation and Directional Emission of Highly Efficient Light Sources. Light: Science \& Applications. 2013; 2:e66.

37. Gabudean AM, Focsan M, Astilean S. Gold Nanorods Performing as Dual-Modal Nanoprobes Via Metal-Enhanced Fluorescence (Mef) and Surface-Enhanced Raman Scattering (Sers). J Phys Chem C. 2012; 116:12240-12249.

38. Lalisse A, Tessier G, Plain J, Baffou G. Quantifying the Efficiency of Plasmonic Materials for near-Field Enhancement and Photothermal Conversion. J Phys Chem C. 2015; 119:25518-25528.

39. Tam F, Goodrich GP, Johnson BR, Halas NJ. Plasmonic Enhancement of Molecular Fluorescence. Nano Lett. 2007; 7:496-501. [PubMed: 17256995]

40. Bakker RM, Yuan HK, Liu Z, Drachev VP, Kildishev AV, Shalaev VM, Pedersen RH, Gresillon S, Boltasseva A. Enhanced Localized Fluorescence in Plasmonic Nanoantennae. Appl Phys Lett. 2008; 92:043101.

41. Stranik O, McEvoy H, McDonagh C, MacCraith B. Plasmonic Enhancement of Fluorescence for Sensor Applications. Sens Actuators, B. 2005; 107:148-153.

42. Fu Y, Zhang J, Lakowicz JR. Plasmonic Enhancement of Single-Molecule Fluorescence near a Silver Nanoparticle. Journal of fluorescence. 2007; 17:811-816. [PubMed: 17922175]

43. Ernst LA, Gupta RK, Mujumdar RB, Waggoner AS. Cyanine Dye Labeling Reagents for Sulfhydryl Groups. Cytometry. 1989; 10:3-10. [PubMed: 2917472]

44. Kelly KL, Coronado E, Zhao LL, Schatz GC. The Optical Properties of Metal Nanoparticles: The Influence of Size, Shape, and Dielectric Environment. J Phys Chem B. 2003; 107:668-677.

45. Bastús NG, Piella J, Puntes V. Quantifying the Sensitivity of Multipolar (Dipolar, Quadrupolar, and Octapolar) Surface Plasmon Resonances in Silver Nanoparticles: The Effect of Size, Composition, and Surface Coating. Langmuir. 2015; 32:290-300. [PubMed: 26649600]

46. Yan R, Gargas D, Yang P. Nanowire Photonics. Nat Photonics. 2009; 3:569-576.

47. Cao L, White JS, Park JS, Schuller JA, Clemens BM, Brongersma ML. Engineering Light Absorption in Semiconductor Nanowire Devices. Nat Mater. 2009; 8:643-647. [PubMed: 19578337]

48. Pasquale AJ, Reinhard BrM, Dal Negro L. Engineering Photonic-Plasmonic Coupling in Metal Nanoparticle Necklaces. Acs Nano. 2011; 5:6578-6585. [PubMed: 21739951] 

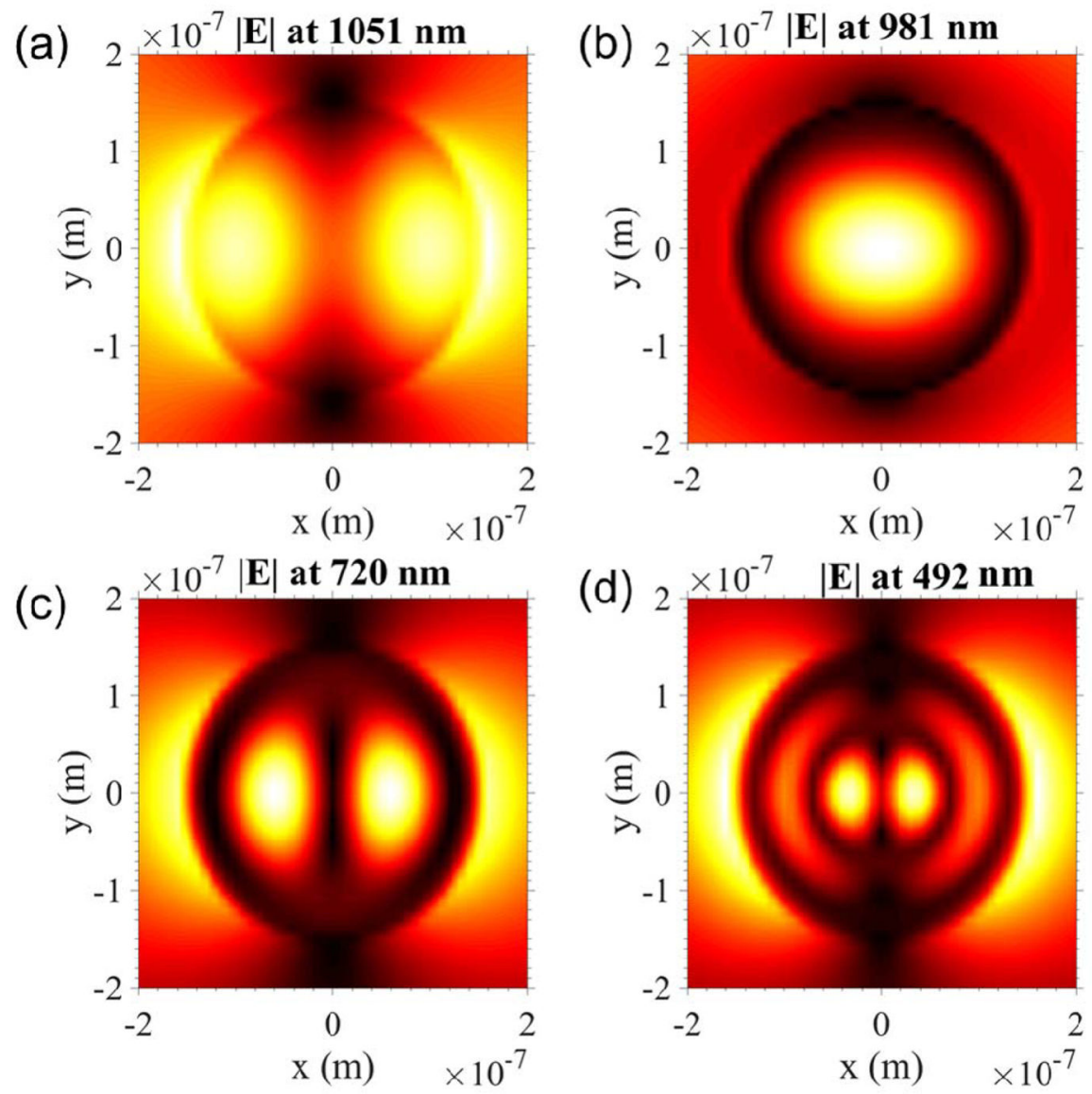

Figure 1.

Simulation results of mode profiles for a silicon nanowire. (a) $\mathrm{HE}_{11}$ of a nanowire with a radius of $150 \mathrm{~nm}$ and length of $3 \mu \mathrm{m}$ excited by an x-polarized incident light. This is the fundamental mode and shows no cutoff. Its signature is the significant modal spillover into the surrounding of the wire. (b-d) $\mathrm{TE}_{01}, \mathrm{HE}_{21}$, and $\mathrm{HE}_{31}$ modes of the same nanowire are higher order modes and are excited at higher frequencies. 


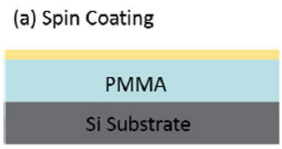

(c) EBL

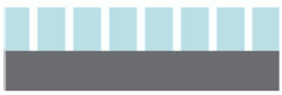

(e) Metal Deposition

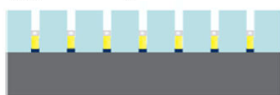

(b) PMMA Lift-off

$\square \mathrm{Al} \approx \mathrm{Au} \square \mathrm{Cr}$

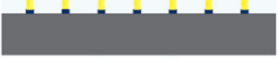

(d) Reactive lon Etching

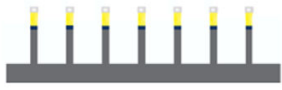

(f) Removal of Al

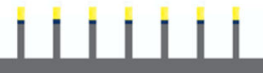

(g) PMMA Lift-off

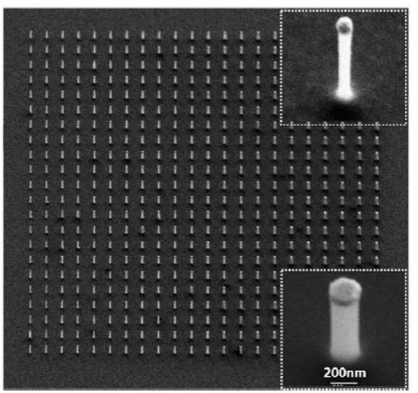

Figure 2.

Fabrication and characterization of NPTWs. (a-f) Schematic illustration of the fabrication process for metallodielectric structures. The hybrid structures were fabricated using EBL followed by RIE. (g) SEM images of the fabricated hybrid structure array. Insets show magnified SEM images of single NPTWs with approximate radii of 50 (top inset) and 100 $\mathrm{nm}$ (bottom inset). 


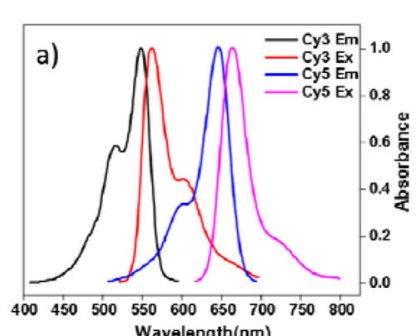

Wavelength(nm)

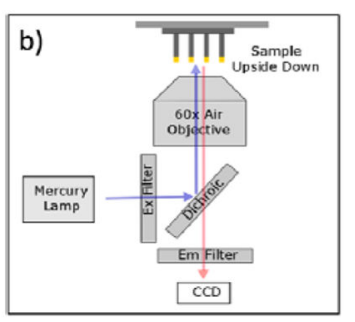

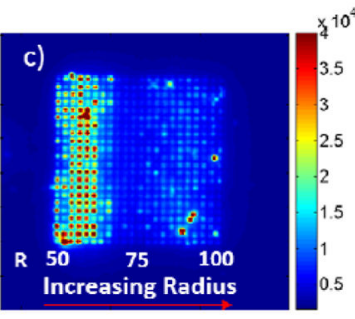

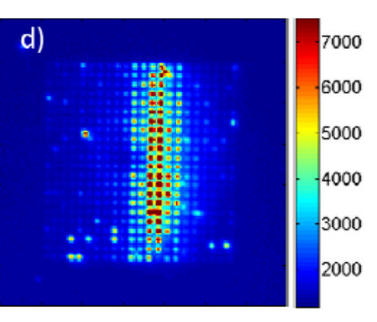

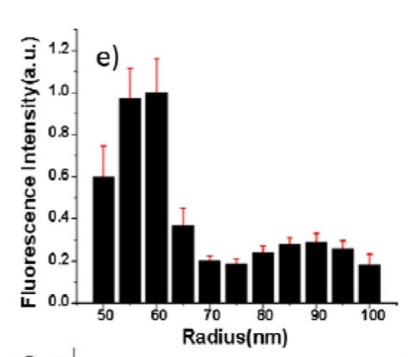

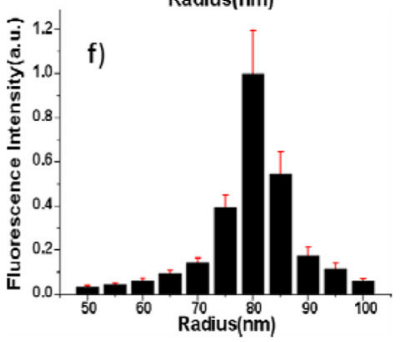

Figure 3.

Radius-dependent fluorescence of dye functionalized NPTWs. (a) Excitation/emission spectra of Cy3/Cy5. (b) Schematic of the fluorescence microscope used for the fluorescence intensity measurement. (c,d) Fluorescence images of hybrid structures with radii ranging from 50 to $100 \mathrm{~nm}$ (with $5 \mathrm{~nm}$ increments) and modified with DNA conjugated Cy3 and $\mathrm{Cy} 5$, respectively. $(\mathrm{e}, \mathrm{f})$ Fluorescence intensity distribution as a function of radius for $\mathrm{Cy} 3$ (e) and $\mathrm{Cy} 5$ (f). 
(a)

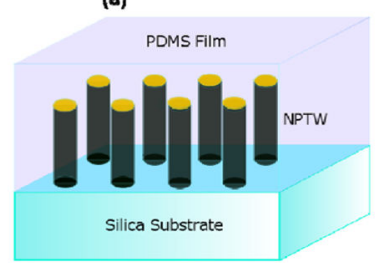

(d) (b)

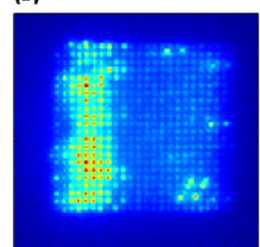

(e) (c)
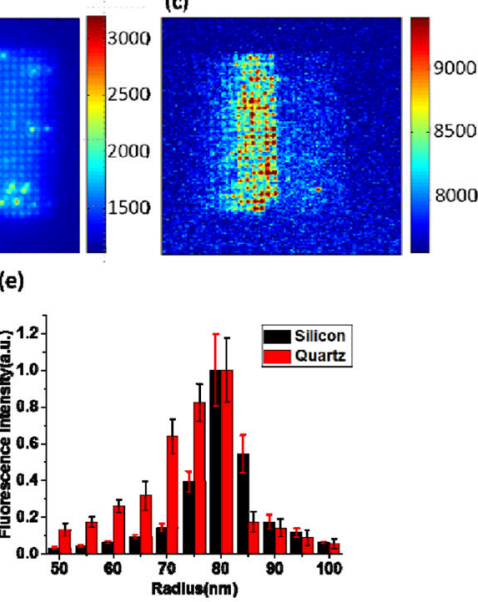

Figure 4.

Fluorescence characterization of PDMS-embedded NPTWs. (a) Scheme of silicon nanowires embedded in PDMS after transfer to a silica substrate. (b,c) Fluorescence images of NPTW arrays embedded in PDMS and modified with Cy3 or Cy5, respectively. (d,e) Comparison of the fluorescence intensity distribution of the function of the radius before/ after PDMS embedding for $\mathrm{Cy} 3$ (d) and Cy5 (e). 
a)

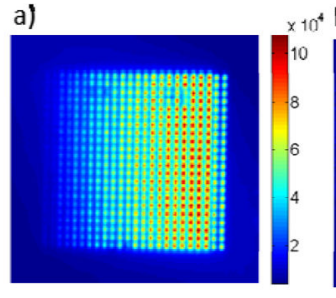

$10^{4}$ b)

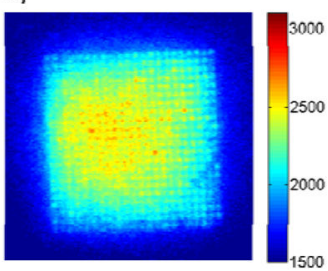

c)

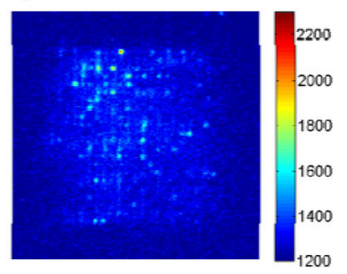

Figure 5.

Characterization of the optical responses from metal nanoparticles in the absence of silicon nanowires. (a) Dark-field image of the gold disk array. (b,c) Fluorescence image of a gold disk array conjugated with $\mathrm{Cy} 3 / \mathrm{Cy} 5$. 
a) $\times 10^{5}$

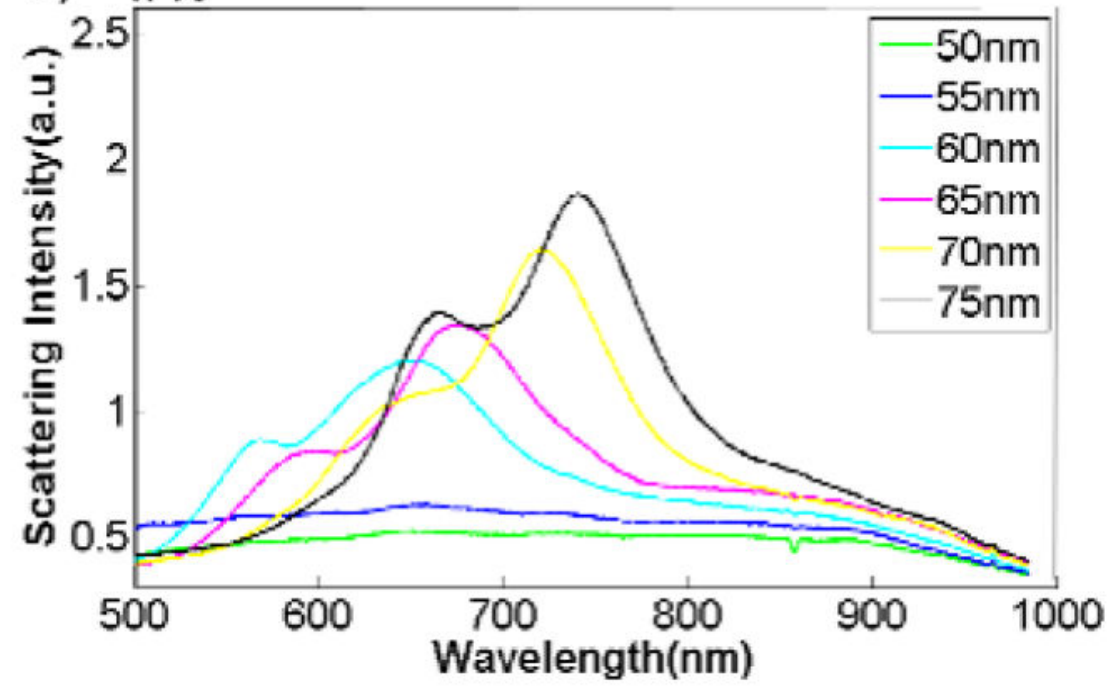

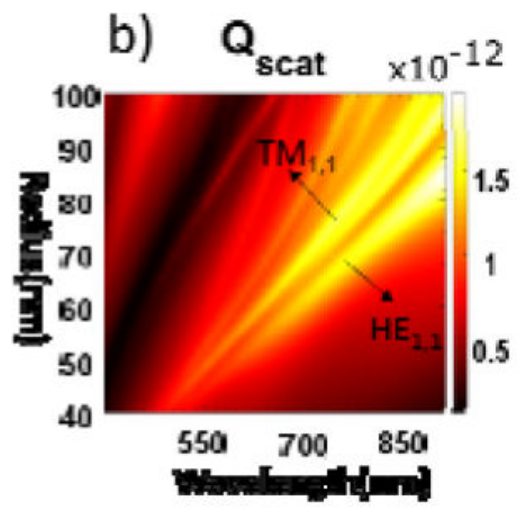

c)

Figure 6.

Scattering spectra of vertical silicon nanowires. (a) Experimental spectra of silicon nanowires. (b) FDTD-simulated scattering spectra of silicon nanowires under oblique incident light illumination. (c) Schematics of the FDTD simulation setup. (d) Measured and simulated spectral peak positions as a function of nanowire radius. 


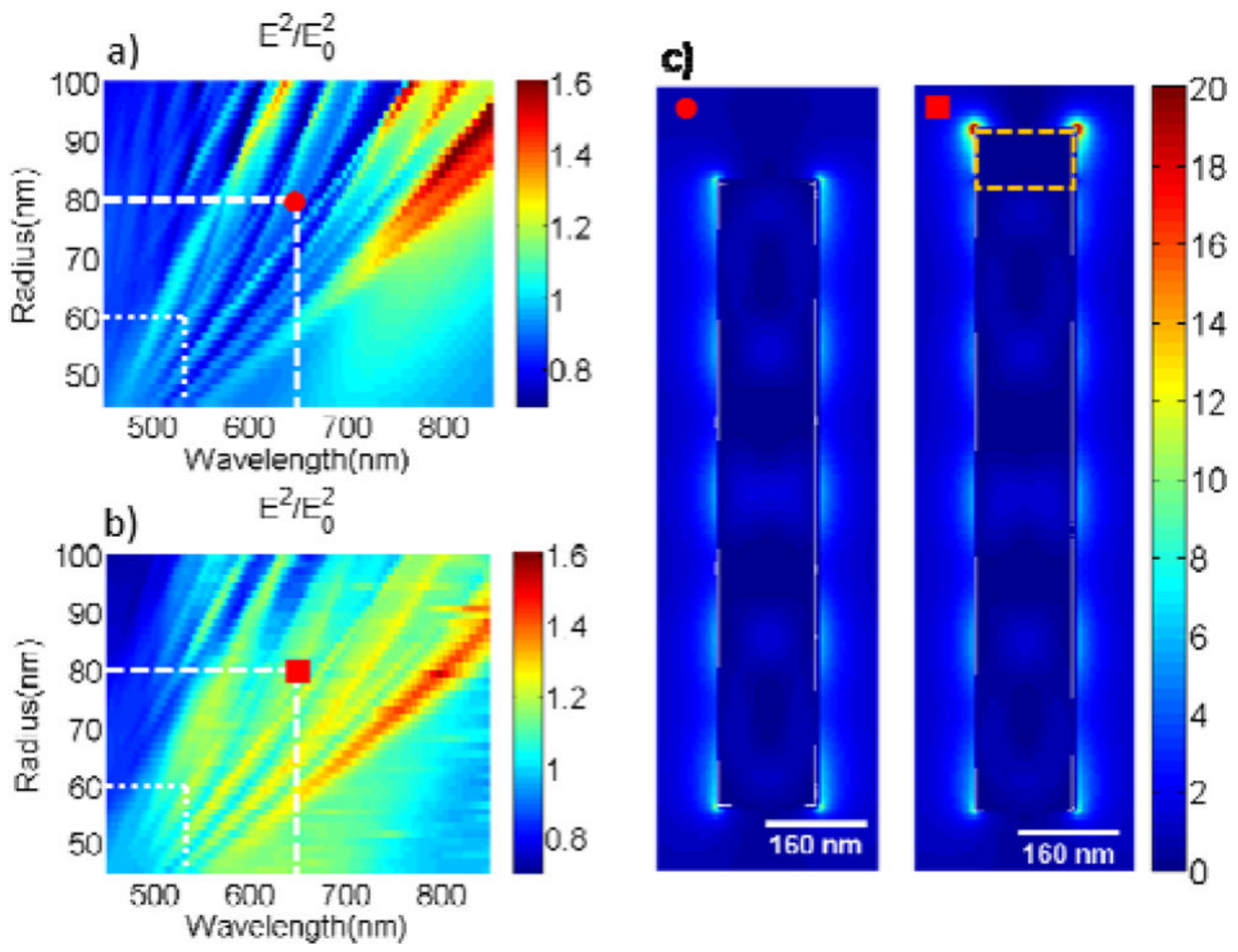

Figure 7.

Near-field properties of the hybrid NPTW system. Simulated near-field intensity spectra of (a) the silicon nanowire only and (b) the hybrid structure with the metal nanoparticle on top of the silicon nanowire. The excitation wavelengths for $\mathrm{Cy} 3$ and $\mathrm{Cy} 5$ are included as dotted and dashed lines, respectively. (c) Near-field distribution in the yz plane for a silicon nanowire (red dot) and NPTW (red square) with the dashed rectangular showing the position of the gold nanoparticle at $\lambda=637 \mathrm{~nm}$. Both wires have a radius of $80 \mathrm{~nm}$. 

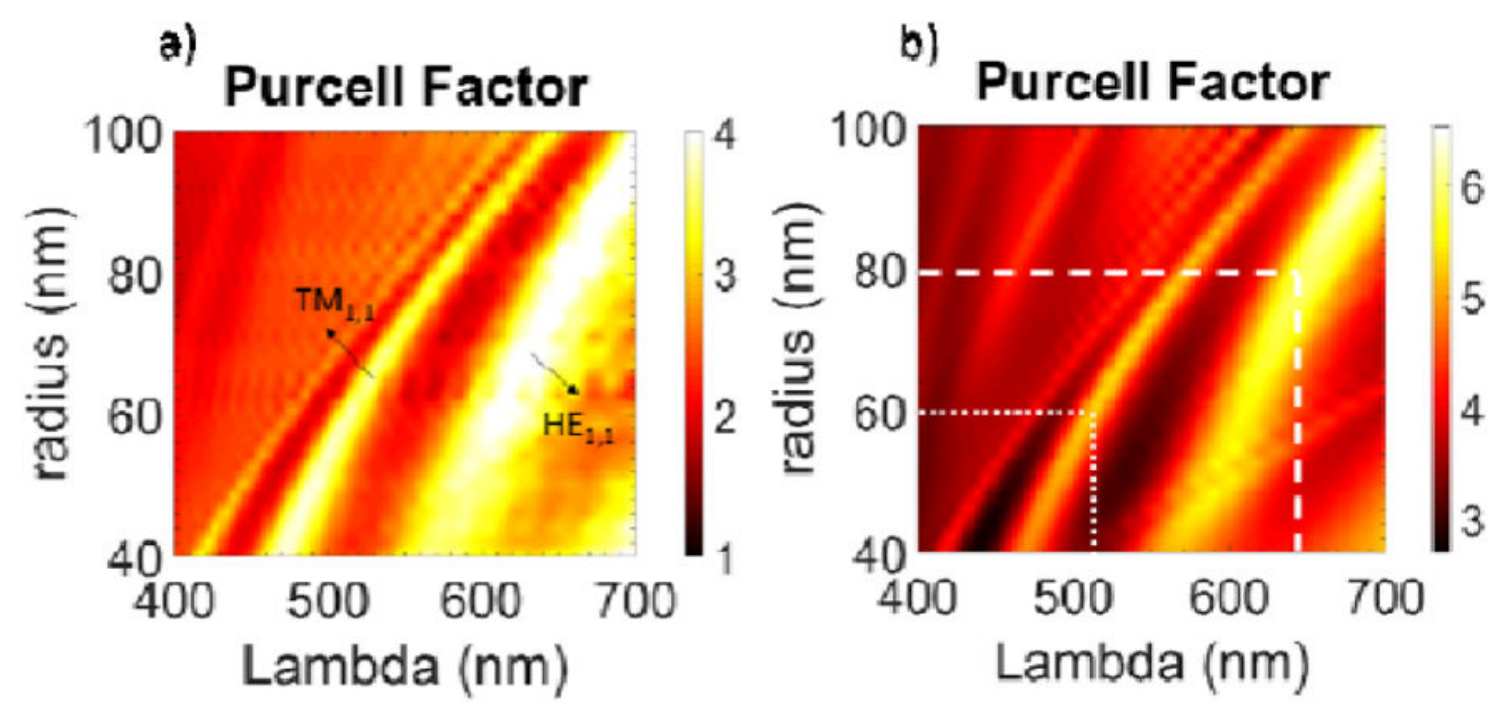

Figure 8.

PF enhancement. Simulation results of PF enhancement for a dipole emitter located (a) onaxis, (b) off-axis. The dipole emitter is oriented with an angle of $45^{\circ}$ with respect to the $\mathrm{z}$ axis. 